OPEN ACCESS

Edited by: Gianpiero Vigani,

University of Turin, Italy

Reviewed by:

Etienne Delannoy,

UMR9213 Institut des Sciences des

Plantes de Paris Saclay (IPS2), France

Jitender Giri,

National Institute of Plant Genome

Research (NIPGR), India

*Correspondence:

Masaru Ohme-Takagi mtakagi@mail.saitama-u.ac.jp

Specialty section: This article was submitted to Plant Nutrition,

a section of the journa

Frontiers in Plant Science

Received: 16 April 2019 Accepted: 24 December 2019

Published: 31 January 2020

Citation:

Yeh C-M, Kobayashi K, Fujii S,

Fukaki $H$, Mitsuda $N$ and Ohme-Takagi M (2020) Blue Light Regulates Phosphate DeficiencyDependent Primary Root Growth Inhibition in Arabidopsis.

Front. Plant Sci. 10:1803. doi: $10.3389 /$ fpls.2019.01803

\section{Blue Light Regulates Phosphate Deficiency-Dependent Primary Root Growth Inhibition in Arabidopsis}

\author{
Chuan-Ming Yeh ${ }^{1,2,3}$, Koichi Kobayashi ${ }^{4}$, Sho Fujii ${ }^{4}$, Hidehiro Fukaki ${ }^{5}$, Nobutaka Mitsuda $^{2}$ \\ and Masaru Ohme-Takagi ${ }^{1,2 *}$
}

${ }^{1}$ Graduate School of Science and Engineering, Saitama University, Saitama, Japan, ${ }^{2}$ Bioproduction Research Institute, National Institute of Advanced Industrial Science and Technology (AIST), Tsukuba, Japan, ${ }^{3}$ Institute of Tropical Plant Sciences and Microbiology, College of Bioscience and Biotechnology, National Cheng Kung University, Tainan, Taiwan, ${ }^{4}$ Graduate School of Arts and Sciences, The University of Tokyo, Tokyo, Japan, ${ }^{5}$ Graduate School of Science, Kobe University, Kobe, Japan

Plants have evolved mechanisms to improve utilization efficiency or acquisition of inorganic phosphate $(\mathrm{Pi})$ in response to Pi deficiency, such as altering root architecture, secreting acid phosphatases, and activating the expression of genes related to Pi uptake and recycling. Although many genes responsive to Pi starvation have been identified, transcription factors that affect tolerance to Pi deficiency have not been well characterized. We show here that the ectopic expression of $B$-BOX32 (BBX32) and the mutation of ELONGATED HYPOCOTYL 5 (HY5), whose transcriptional activity is negatively regulated by BBX32, resulted in the tolerance to $\mathrm{Pi}$ deficiency in Arabidopsis. The primary root lengths of 35S:BBX32 and hy5 plants were only slightly inhibited under Pi deficient condition and the fresh weights were significantly higher than those of wild type. The $\mathrm{Pi}$ deficiency-tolerant root phenotype of hy5 was similarly observed when grown on the medium without $\mathrm{Pi}$. In addition, a double mutant, hy5 s/r1, without lateral roots, also showed a long primary root phenotype under phosphate deficiency, indicating that the root phenotype of hy5 does not result from an increase of external Pi uptake. Moreover, we found that blue light may regulate $\mathrm{Pi}$ deficiency-dependent primary root growth inhibition through activating peroxidase gene expression, suggesting the Pi-deficiency tolerant root phenotype of hy5 may be due to blockage of blue light responses. Altogether, this study points out light quality may play an important role in the regulation of $\mathrm{Pi}$ deficiency responses. It may contribute to regulate plant growth under Pi deficiency through proper illumination.

Keywords: BBX32, HY5, light, phosphate deficiency, root architecture, transcription factor

\footnotetext{
Abbreviations: B, blue; BBX32, B-BOX32; DGDG, digalactosyldiacylglycerol; FR, far-red; hps, hypersensitive to phosphate starvation; HY5, ELONGATED HYPOCOTYL 5; LR, lateral root; MGDG, monogalactosyldiacylglycerol; NPC4, non-specific phospholipase C4; PC, phosphatidylcholine; PHL1, PHR1-like 1; PHO1, PHOSPHATE1; PHR1, PHOSPHATE STARVATION RESPONSE 1; Pi, inorganic phosphate; PR, primary root; PSI, phosphate starvation-induced; PSR, phosphate starvation response; R, red; slr-1, solitary-root-1; SQDG, sulfoquinovosyldiacylglycerol; TF, transcription factor; WT, wild type.
} 


\section{INTRODUCTION}

Inorganic phosphate $(\mathrm{Pi})$ is an essential constituent of ATP, nucleic acids, and membrane phospholipids. In addition, it is crucial to various cellular metabolic pathways, including photosynthesis, glycolysis, respiration, signal transduction, and carbohydrate metabolism (Ticconi and Abel, 2004; Péret et al., 2011; Niu, 2013). However, Pi is easily chelated by soil particles or formed insoluble complexes with aluminum or iron at acid $\mathrm{pH}$ and with calcium at alkaline $\mathrm{pH}$ leading to a low mobility and availability in soils (Wissuwa, 2003; Gaxiola et al., 2011). Therefore, available soil Pi concentrations are often less than the requirement for optimal crop production (Nussaume et al., 2011; Péret et al., 2011; Niu, 2013). Plants have evolved adaptive mechanisms to acquire and recycle $\mathrm{Pi}$ in response to Pi deficiency. Alteration of root architecture, such as enhancement of lateral root growth and root hair formation, increases root surface areas for $\mathrm{Pi}$ absorption (Ticconi and Abel, 2004; Péret et al., 2011). Induction of high-affinity $\mathrm{Pi}$ transporter genes increases uptake of soluble $\mathrm{Pi}$, while activation or secretion of acid phosphatases, ribonucleases, and organic acids enhances scavenging of extracellular Pi from insoluble organic complexes. In addition, the activities of acid phosphatases and ribonucleases also help release Pi from intracellular organic Pi-containing molecules (Raghothama, 2000; Poirier and Bucher, 2002; Nussaume et al., 2011). To improve Pi use efficiency, plants substitute bypass pathways that do not require $\mathrm{Pi}$ for metabolic processes requiring Pi (Plaxton and Tran, 2011). Replacing membrane phospholipids with non-P-containing glycolipids also plays an important role in the supply of free $\mathrm{Pi}$ during Pi deficiency (Kobayashi et al., 2006).

Many efforts have been made to unravel the molecular mechanisms that regulate Pi starvation responses (PSRs). An array of Pi starvation-induced (PSI) genes have been identified by transcriptome studies (Wu et al., 2003; Misson et al., 2005; Thibaud et al., 2010; Woo et al., 2012) and a series of hypersensitive to phosphate starvation ( $h p s$ ) mutants have been isolated and characterized (Yeh et al., 2017). Although various plant transcription factors (TFs) affect PSRs, the transcriptional regulation of these processes is not yet well elucidated. AtPHR1 (PHOSPHATE STARVATION RESPONSE 1) is the first Arabidopsis TF gene shown to mediate diverse PSRs (Rubio et al., 2001). Although AtPHR1 is not Pi starvation-inducible, PHR1 regulates a subset of PSI genes through the miR399-PHO2 (an ubiquitin-conjugating E2 enzyme) signaling pathway (Bari et al., 2006; Chiou et al., 2006). AtPHR1, AtPHL1 (PHR1-like 1), and their two rice orthologues, OsPHR1 and OsPHR2, have been identified as having partially redundant functions (Zhou et al., 2008; Bustos et al., 2010; Liu et al., 2010). In addition, several TFs have been identified as negative regulators of PSRs in Arabidopsis. BHLH32, a basic helix-loop-helix TF, negatively regulates anthocyanin accumulation, root hair formation, and induction of the PSI genes (Chen et al., 2007). AtMYB62 is lowPi-inducible and mediates its negative effects on PSRs through modulation of gibberellin metabolism (Devaiah et al., 2009). WRKY6 and WRKY42 negatively regulate the expression of PHOSPHATE1 (PHO1), which is responsible for Pi translocation from root to shoot in Arabidopsis (Hamburger et al., 2002; Chen et al., 2009). AtWRKY75 and AtZAT6 have been reported to regulate root development and $\mathrm{Pi}$ acquisition, although they may not be specific to PSRs due to their responsiveness to multiple nutrient deficiencies (Devaiah et al., 2007a; Devaiah et al., 2007b). In recent years, several Arabidopsis TF genes, such as AtERF070, APSR1, AtMYB2, and AL6, have been shown to be involved in the regulation of root growth and architecture under Pi deficiency (Yeh and Ohme-Takagi, 2015).

Adding Pi fertilizer can improve soil Pi levels; however, the world's Pi rock reserves may be exhausted within 120 years (Gilbert, 2009; Nussaume et al., 2011) and the demand for Pi fertilizers will likely increase to support crop productivity for the growing global population (Nussaume et al., 2011; Péret et al., 2011). In addition, the low solubility of Pi in soils often causes over-application of chemical fertilizers, subsequently leading to potential threats to the environment and the ecosystem (Gaxiola et al., 2011; Péret et al., 2011). Therefore, proper utilization of the remaining Pi reserves is important to reduce Pi resource depletion and environmental threaten. To this end, development of crops with tolerance to $\mathrm{Pi}$ deficiency is required, especially if crops can be manipulated to possess higher ability for $\mathrm{Pi}$ recycling or $\mathrm{Pi}$ utilization efficiency.

In this study, we identified both the chimeric repressor and the ectopic expression of a B-box zinc finger protein (BBX32) gene (35S:BBX32-SRDX and 35S:BBX32) induce Pi deficiency tolerance and the $\mathrm{Pi}$-deficiency tolerance phenotype of $35 \mathrm{~S}$ : $B B X 32-S R D X$ and 35S:BBX32 may result from the repression of HY5 function. Furthermore, we found that continuous blue light illumination accelerates sensitivity to Pi deficiency in WT and elimination from blue light improves $\mathrm{PR}$ growth through reduction of class III peroxidase (PRX) gene expression. Our results indicate that $\mathrm{BBX} 32$ and $\mathrm{HY} 5$ are involved in the regulation of PSRs, and 35S:BBX32-SRDX and hy5 exhibit root tolerant phenotypes to Pi deficiency due to blockage of bluelight responses.

\section{RESULTS AND DISCUSSION}

\section{Identification of Transcription Factors Responsible for Pi Deficiency Tolerance}

To identify transcription factors (TFs) that can be manipulated to allow plants growing well under minimal Pi fertilization, we grew the Arabidopsis Chimeric REpressor gene Silencing Technology (CRES-T) lines in Pi-deficient conditions and screened for plant phenotypes indicative of tolerance to $\mathrm{Pi}$ deficiency: larger plant size, longer primary root (PR) length, and lower anthocyanin accumulation than wild type (WT). The CRES-T can convert a transcriptional activator into a strong repressor by fusion with the plant-specific repression domain SRDX (superman repression domain $\mathrm{X}$ ), leading to dominant repression of the target genes. The resultant transgenic plants exhibit phenotypes similar to those of the knockout mutants of the manipulated TF and its functionally redundant TFs (Hiratsu et al., 2003; Mitsuda and Ohme-Takagi, 2009). In this study, Arabidopsis CRES-T seedlings were grown on 1/2 MS medium 
with $10 \mu \mathrm{M} \mathrm{Pi}\left(\mathrm{K}_{2} \mathrm{HPO}_{4}\right)$ to isolate plants that show tolerance to Pi deficiency. A CRES-T line for BBX32 gene (35S:BBX32-SRDX) was isolated and preferentially investigated.

The 35S:BBX32-SRDX lines and the corresponding WT, Col0 , were grown on $1 / 2 \mathrm{MS}$ media in the presence of $625 \mu \mathrm{M} \mathrm{Pi}(\mathrm{Pi}$ sufficient) or $10 \mu \mathrm{M} \mathrm{Pi}$ (Pi deficient) for 10 days to confirm the tolerant phenotype. The PR growth of both WT and 35S:BBX32$S R D X$ was inhibited under Pi deficient condition compared to $\mathrm{Pi}$ sufficient condition, while the PR lengths of 35S:BBX32-SRDX were significantly longer than that of WT (Figures 1A, B). In addition, the fresh weight of 35S:BBX32-SRDX under $\mathrm{Pi}$ deficiency was higher compared to the WT (Figure 1C and Supplementary Figure 1). The Pi-deficiency tolerance of 35S: $B B X 32-S R D X$ was further confirmed by examination of $\mathrm{Pi}$ deficiency-responsive anthocyanin accumulation and gene expression. The anthocyanin content was increased in both $\mathrm{WT}$ and 35S:BBX32-SRDX under Pi-deficient conditions; however, the level was significantly lower in 35S:BBX32-SRDX than in WT (Figure 2A). The expression of INDUCED BY PHOSPHATE STARVATION1 (IPS1), RIBONUCLEASE 1 (RNS1), and ACID PHOSPHATASE TYPE 5 (ACP5) was significantly induced by $\mathrm{Pi}$ deficiency in the WT, but their transcript levels were much lower in 35S:BBX32-SRDX than in the WT (Figures 2B-D). These results indicate that 35S:BBX32$S R D X$ plants appear to suffer less stress in $\mathrm{Pi}$-deficient conditions compared to the WT.

\section{Phenotype of Overexpression and Knockout Lines of BBX32}

To further examine the role of $B B X 32$ in response to Pi deficiency, the overexpression lines of $B B X 32$ (35S:BBX32) and $b b \times 32$ knockout plants (SALK_059534) were generated and examined. The 35S: $B B X 32$ plants exhibited a Pi deficiency-tolerant phenotype as $35 S$ : $B B X 32-S R D X$ plants (Figures 3A, B), while the inhibition of $\mathrm{PR}$ length of $b b \times 32$ under Pi deficiency was similar to that of WT (Figure 3C). As shown previously, when a TF is a repressor or inhibitor of transcription, the chimeric repressor and the ectopic expression of a TF exhibit a similar phenotype but show opposite to the knockout line (Matsui et al., 2008; Ikeda et al., 2012). These indicate that BBX32 is likely to play a role in negative regulation in response to $\mathrm{Pi}$ deficiency.

\section{Tolerant Phenotypes of hy5-215 Mutants Under Pi Deficiency}

BBX32 has been reported to modulate light signaling through suppressing activity of ELONGATED HYPOCOTYL 5 (HY5), a

A
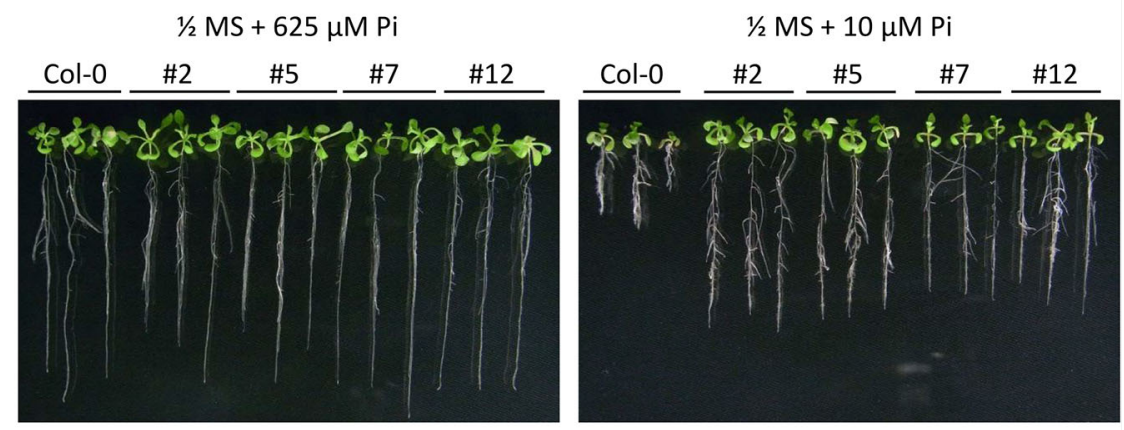

B

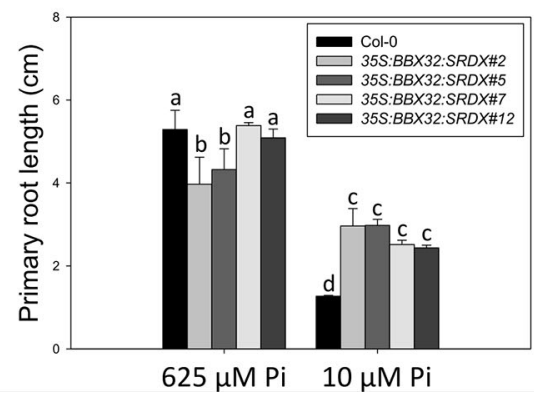

$\mathrm{C}$

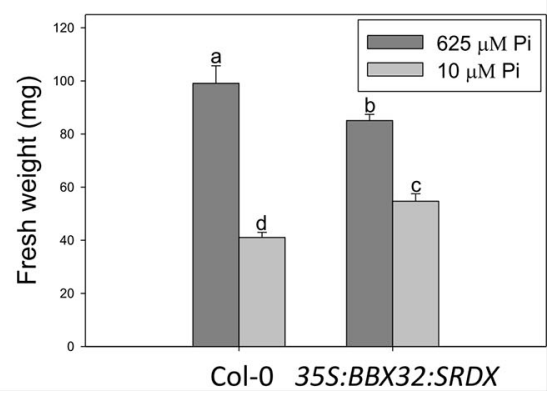

FIGURE 1 | Primary root length and fresh weight of wild-type and 35S:BBX32-SRDX seedlings in response to Pi treatment. (A) Wildtype (Col-0) and 35S:BBX32SRDX seedlings (line\#2, 5, 7, 12) grown in Pi-sufficient $(625 \mu \mathrm{M})$ and Pi-deficient $(10 \mu \mathrm{M})$ conditions. (B) Primary root (PR) lengths after growth on vertical plates for 10 days. (C) Seedling fresh weights (FWs) after growth on horizontal plates for 16 days. The FW of 35S:BBX32-SRDX is the average of the four lines used in this study. Data represent the means \pm standard error (SE) of four independent experiments. Different letters above the bars indicate statistically significant differences among the means based on Two-way ANOVA (Analysis of Variance) followed by Fisher's LSD (Least Significant Difference) tests $(P<0.05)$. 
A

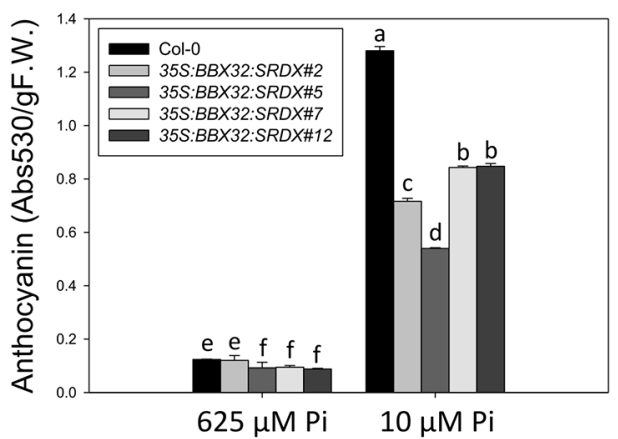

C

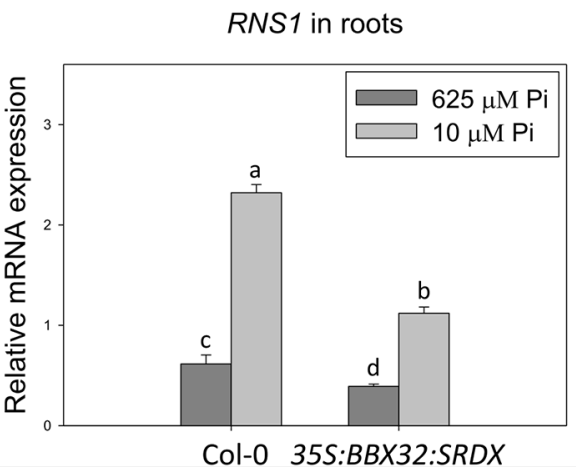

B

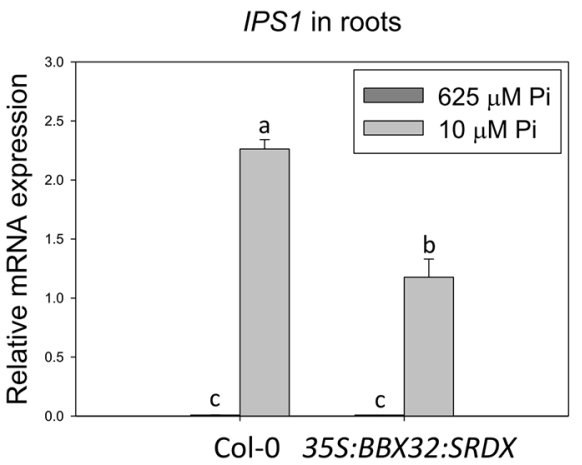

$\mathrm{D}$

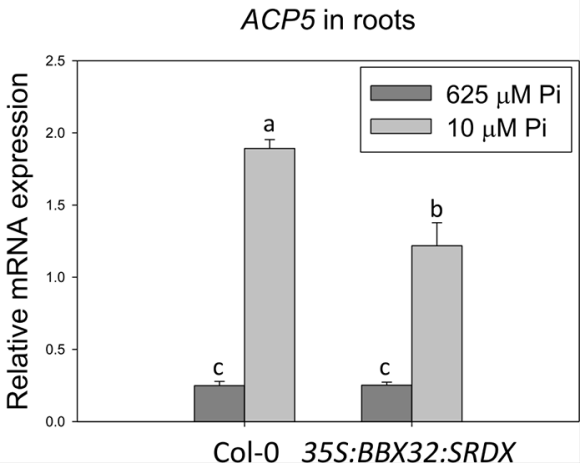

FIGURE 2 | Lower level of anthocyanin content and PSI gene expression in 35S:BBX32-SRDX plants. Low Pi-induced anthocyanin accumulation in Col-0 and 35S: BBX32-SRDX (A). Expression level of IPS1 (B), RNS1 (C), and ACP5 (D). Anthocyanin content of 10-day-old seedlings was extracted by $45 \%$ methanol and 5\% acetic acid. The relative level of anthocyanin was calculated from the absorbance at 530 and $637 \mathrm{~nm}$. RNA extracted from 10-day-old seedlings was subjected to real-time RT-PCR. Data represent means \pm standard error (SE) of three independent experiments. Different letters above the bars indicate statistically significant differences among the means based on Two-way ANOVA followed by Fisher's LSD test $(P<0.05)$.

bzip TF involved in the regulation of photomorphogenesis. Overexpression of $B B X 32$ induces hypocotyl elongation in response to multiple wavelengths of light as in hy5 mutant and represses HY5-regulated gene expression (Holtan et al., 2011). Therefore, we examined whether hy5 mutant also exhibits $\mathrm{Pi}$ deficiency-tolerant phenotype. Here, we used hy5-215, generated by ethyl methanesulfonate mutagenesis, for further study (Oyama et al., 1997). The PR lengths of WT were significantly reduced under Pi-deficient conditions when compared with those grown under Pi-sufficient conditions, while only slight inhibition of $\mathrm{PR}$ growth was observed in the hy5-215 mutant between Pisufficient and Pi-deficient conditions (Figures 4A, B). WT fresh weight declined to $37 \%$ under Pi deficient-conditions compared to Pi-sufficient conditions, while hy5-215 fresh weight declined to 65\% under Pi deficient-conditions compared to Pi-sufficient conditions (Figure 4C and Supplementary Figure 2). We also confirmed the tolerance of $h y 5-215$ to Pi deficiency by examination of several wellknown PSRs including expression of ribonuclease, purple acid phosphatase, and anthocyanin biosynthesis genes (Supplementary
Note and Figures 3-5). These results indicate that the Pi-deficiency tolerant phenotype of 35S:BBX32-SRDX and 35S:BBX32 plants may indeed result from repression of HY5 function. We, therefore, would use hy5-215 mutant for further studies to address the tolerance mechanisms.

\section{Alteration of Root Architecture in hy5-215 Is Not Responsible to Pi-Deficiency Tolerance}

Plant root architecture, the spatial arrangement of a root system, is highly plastic in response to depletion of mineral nutrients. Modifications of RA through altering the number, length, angle, and diameter of roots or root hairs enable plants to cope with nutrient shortages (Gruber et al., 2013). The "topsoil foraging" strategy is employed to get immobile $\mathrm{Pi}$ from the Pi-enriched upper-layer soil under Pi deficiency; in topsoil foraging, plants inhibit PR growth but enhance lateral root (LR) growth and root hair formation, thus increasing the surface area available for $\mathrm{Pi}$ uptake (Péret et al., 2011; Sato and Miura, 2011; Niu, 2013). In 
A



B

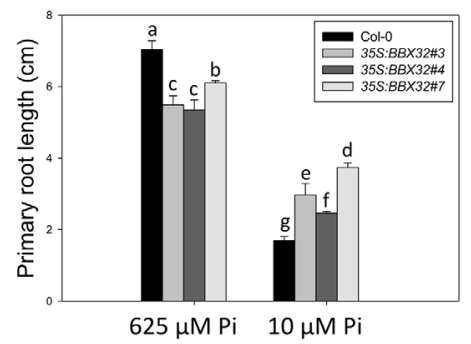

$\mathrm{C}$

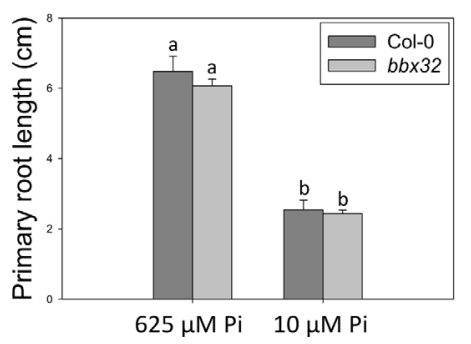

FIGURE 3 | Primary root length of 35S:BBX32 and bbx32 seedlings in response to Pi treatment. (A) Wildtype and 35S:BBX32 seedlings (line\#3, 4, 7) grown in Pi-sufficient and Pi-deficient conditions. (B) PR lengths of $35 S$ : BBX32 after growth on vertical plates for 10 days. (C) PR length of $b b \times 32$ after growth on vertical plates for 10 days. Data represent the means \pm standard error (SE) of four independent experiments. Different letters above the bars indicate statistically significant differences among the means based on Two-way ANOVA followed by Fisher's LSD test $(P<0.05)$.

this study, a great number of root hairs were initiated in the WT under Pi-deficient conditions, whereas hy5-215 formed fewer and shorter root hairs (Figure 5A), suggesting that hy5-215 may not show as strong of a response to Pi deficiency as WT. Bhosale et al. (2018) reported that Arabidopsis root hair elongation in response to low $\mathrm{Pi}$ is regulated by an auxin-dependent pathway mediated by TAA1, AUX1, ARF19, RSL2, and RSL4. We analyzed expression levels of these genes in the roots of WT and hy5-215 and found no significant difference between WT and mutant (data not shown), suggesting the hy5-215 phenotype doesn't result from suppression of root hair elongation. It is worth noting that LR numbers and lengths were not enhanced by low-Pi treatment in either WT or hy5-215. Instead, LR growth was repressed by our Pi deficiency condition (Figures 5B-D). This inconsistency to previous findings may result from different Pi concentrations and experimental conditions used in the different studies. Plants grown at relatively higher levels of $\mathrm{Pi}$ $(>1 \mathrm{mM})$ in Pi-sufficient media form fewer or almost no LRs (Pérez-Torres et al., 2008; Lei et al., 2011). However, Pi-sufficient treatment $(625 \mu \mathrm{M})$ in this work induces much more LR formation and growth. This is in agreement with the previous reports that used relative lower concentrations for Pi-sufficient treatments (Devaiah et al., 2007a; Pérez-Torres et al., 2008; Devaiah et al., 2009; Lei et al., 2011; Gruber et al., 2013).

Although LR growth was not enhanced by Pi starvation in this study, a root system possessing more and longer LRs was found in hy5-215 in both Pi-sufficient and Pi-deficient conditions (Figures 5B-D). A similar phenotype was found in 35S:BBX32$S R D X$ (Supplementary Figure 6). To examine whether the increased LR number and lengths contribute to the Pideficiency tolerance in hy5-215, a double mutant constructed with hy5-215 and solitary-root-1 (slr-1), a gain-of-function mutant of IAA14 (a repressor of auxin signaling) that does not produce LRs, was examined under Pi deficiency (Fukaki et al., 2002; Kobayashi et al., 2012). The hy5-215 slr-1 double mutant showed a long-hypocotyl phenotype similar to that of hy5-215 and a PR lacking LR growth similar to the slr-1 phenotype. Interestingly, the PR elongation of hy5-215 slr-1 seedlings was only slightly inhibited by Pi deficiency, although the PR of hy5215 slr-1 was shorter than that of hy5-215 in the respective conditions (Figures 5E, F). The results revealed that LR growth is beneficial for growth on Pi-deficient medium, but the change in hy5-215 root architecture does not appear to be responsible for the observed tolerance to $\mathrm{Pi}$ deficiency in hy5-215. Auxin signaling was reported to be enhanced in Arabidopsis hy5 mutants (Oyama et al., 1997; Cluis et al., 2004), whereas it may be repressed in hy5-215 slr-1 mutants due to the gain-of-function mutation of SLR/IAA14. Therefore, the similar tolerance phenotypes between hy5-215 slr-1 and hy5-215 also suggest that auxin signaling may not be responsible for the $\mathrm{Pi}$ deficiency tolerance in hy5-215.

\section{External Pi Acquisition Is Not Involved in Pi-Deficiency Tolerance of hy5-215}

Enhancement of Pi influx through induction of high-affinity $\mathrm{P}$ transporter genes is one of the conserved strategies evolved by plants to optimize their growth in response to Pi limitation. There are nine genes encoding PHT homologs (PHT1;1PHT1;9) in the Arabidopsis genome. Most of the PHT1 family genes are strongly induced by low Pi treatment within the first 12 hours (Bayle et al., 2011; Nussaume et al., 2011). Functional studies show a major role for PHT1 in Pi acquisition in roots from Pi-deficient environment; however, some of the PHTs are also required for Pi mobilization (PHT1;5, PHT1;8 and PHT1;9), flower development $(\mathrm{PHT} 1 ; 6)$, and $\mathrm{Pi}$ uptake in $\mathrm{Pi}$ replete 
A

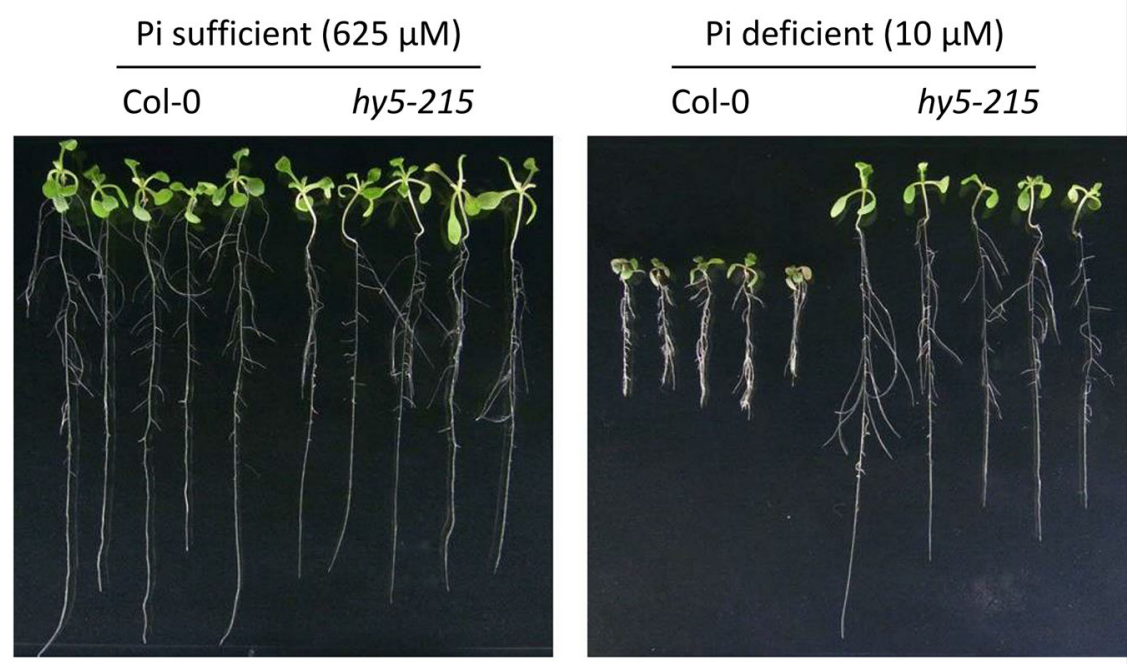

B

C
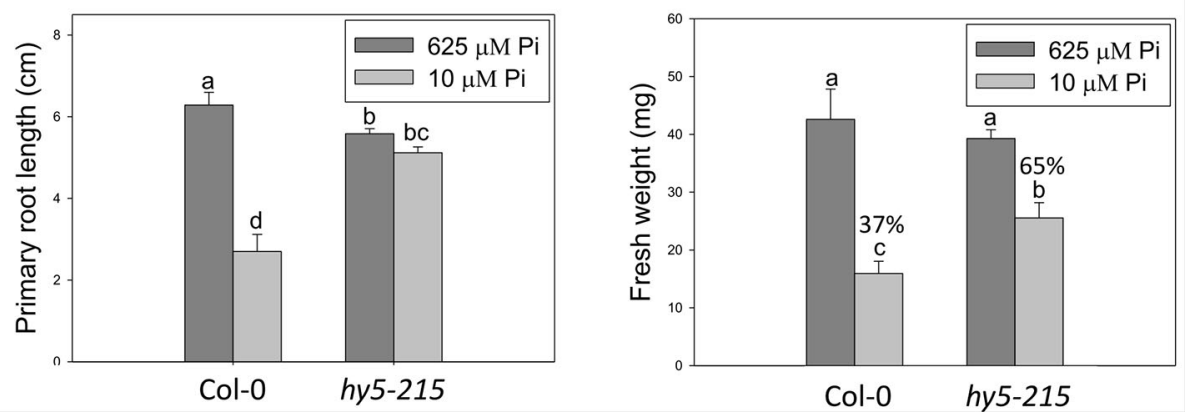

FIGURE 4 | Primary root length and fresh weight of wild-type and mutant seedlings in response to Pi treatment. (A) Wildtype and hy5-215 seedlings grown in Pisufficient and Pi-deficient conditions. (B) PR lengths after growth on vertical plates for 10 days. (C) Seedling FWs after growth on horizontal plates for 14 days. Data represent the means \pm standard error (SE) of four independent experiments. Different letters above the bars indicate statistically significant differences among the means based on Two-way ANOVA (Analysis of Variance) followed by Fisher's LSD (Least Significant Difference) tests $(P<0.05)$.

condition (PHT1;1 and PHT1;4) (Nussaume et al., 2011; Nagarajan et al., 2011; Lapis-Gaza et al., 2014). In this study, we found that expression of PHT1 family genes was lower in hy5215 shoots than in the WT, suggesting hy5-215 may not be as deficient as WT under low Pi treatment (Supplementary Figure 7). However, three PHT1 genes, PHT1;5, PHT1;8, and PHT1;9, were induced in a higher level in hy5-215 roots under both sufficient and deficient conditions (Supplementary Figure 8 and Table 1). To demonstrate whether the higher PHT1 gene expression in hy5-215 roots can increase $\mathrm{Pi}$ uptake and subsequently contribute to Pi-deficiency tolerance, the free $\mathrm{Pi}$ content was measured. Unexpectedly, a great reduction of $\mathrm{Pi}$ level was found in hy5-215 shoots under Pi sufficient condition (Figure 6A). Although Pi content was slightly higher in hy5-215 shoots than in WT under Pi deficiency, no statistically significant difference $(P<0.05)$ was found between them. In addition, there was no significant difference between WT and hy5-215 in roots (Figure 6B). The results indicated that the elevated amounts of PHT1 transcripts in hy5-215 roots might not or only partially contribute to Pi deficiency tolerance of hy5-215. To verify this finding, we cultured WT and hy5-215 plants on Pi-free media. The hy5-215 plants exhibited similar growth on Pi-free medium and on Pi-deficient medium containing $10 \mu \mathrm{M}$ Pi. The PR length of hy5-215 grown on Pi-free medium was only slightly diminished compared to that of plants grown on Pi-deficient medium (Figure 6C and Supplementary Figure 9A). A similar phenotype was found in 35S:BBX32-SRDX (Supplementary Figure 9B). Altogether, these results indicate that the induction of PHT1;5, PHT1;8, and PHT1;9 in hy5-215 roots may be involved in Pi translocation from roots to shoots but not Pi uptake from roots, and the root tolerant phenotype of hy5-215 to $\mathrm{Pi}$ deficiency is not related to extracellular $\mathrm{Pi}$ acquisition. 
A

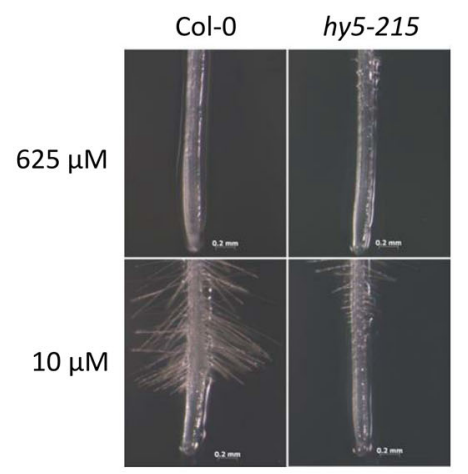

$\mathrm{C}$

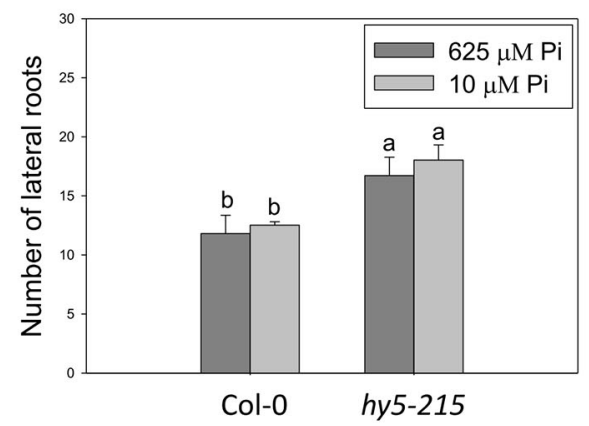

$\mathrm{E}$

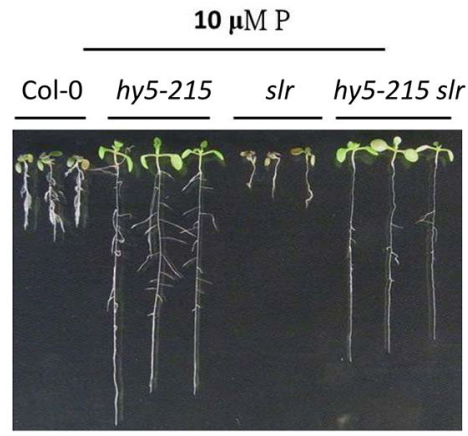

B

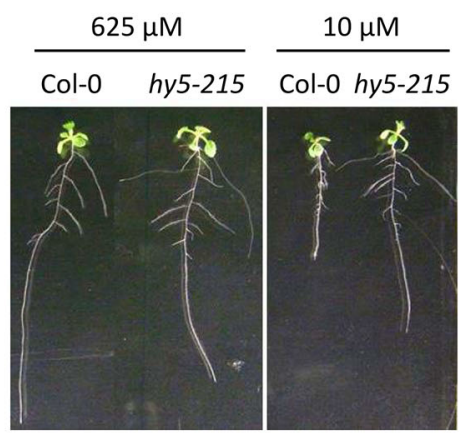

$\mathrm{D}$

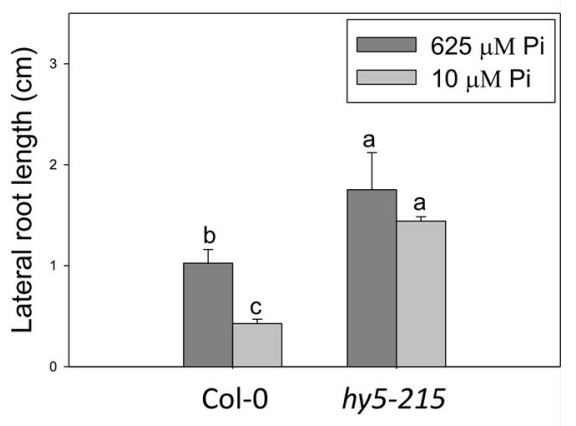

F

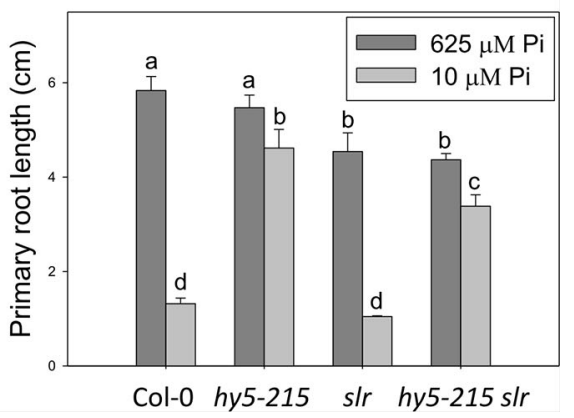

FIGURE 5 | Root hair formation and root architecture of wild-type and mutant seedlings in response to Pi treatment. (A) Root hair formation of Col-0 and hy5-215 after growth of 7 days. (B) Root architecture of Col-0 and hy5-215 after growth of 10 days. (C) Increase of LR number in hy5-215 plants. (D) Increase of LR length in hy5-215 plants. (E) The Col-0, hy5-215, slr, and hy5-215slr-1 seedlings grown in Pi-sufficient and Pi-deficient conditions. (F) PR length in Col-0, hy5-215, slr, and hy5-215s/r-1. All the seedlings were grown on $1 / 2 \mathrm{MS}$ medium with 625 or $10 \mu \mathrm{M}$ Pi for 7 to 10 days. Data represent means \pm SE of four independent experiments. Different letters above the bars indicate statistically significant differences among the means based on Two-way ANOVA followed by Fisher's LSD tests $(P<0.05)$.

Furthermore, it also suggests that the pre-accumulated $\mathrm{Pi}$ in seeds during seed development is sufficient to support hy5-215 growth at the early stages of Pi deficiency.

\section{Lower Level of Pi Deficiency-Inducible Membrane Glycolipids in hy5-215}

Since Pi deficiency tolerance of hy5-215 was not due to Pi acquisition, we investigated Pi use efficiency in the mutant and wild type. Improvement of Pi utilization efficiency helps plants to conserve internal $\mathrm{Pi}$ and can involve the recycling of Pi from senescent tissues and the replacement of $\mathrm{Pi}$ from cellular structures or metabolic processes by alternative non-Pi compounds (Kobayashi et al., 2006; Rose et al., 2013). Membrane lipid remodeling, in which phospholipids are hydrolyzed and replaced by non-phosphorus glycolipids, such as sulfoquinovosyldiacylglycerol (SQDG) and 
digalactosyldiacylglycerol (DGDG), is a representative mechanism of Pi recycling, which improves Pi use efficiency (Kobayashi et al., 2006; Nakamura, 2013). Therefore, we analyzed the expression of genes involved in hydrolysis of phospholipids, non-specific phospholipase C4 gene (NPC4), and synthesis of SQDG and DGDG including SQD1, SQD2, $M G D G 2$, and MGDG3 (monogalactosyldiacylglycerol synthetic genes) in the WT and hy5-215. All the analyzed genes were induced by Pi deficiency, but the expression levels were lower in hy5-215 than in the WT (Supplementary Figures 10A-E). The lipid composition calculated as the ratio of DGDG and PC (phosphatidylcholine), one of the major membrane phospholipids, is used as a marker to indicate a Pi-deficient state (Kobayashi et al., 2006). Enhancement of the DGDG/PC ratio represents an increase in DGDG biosynthesis to replace membrane phospholipids in response to Pi deficiency. A lower ratio of DGDG/PC was found in hy5-215 under Pi-deficient conditions (Supplementary Figure 10F), indicating that the increased tolerance to Pi deficiency in hy5-215 mutants is not caused by increased free Pi from phospholipids.

\section{Light Quality Is Involved in Regulation of Pi Deficiency Response}

Because HY5 acts as an integrator of different light signaling pathways downstream of multiple photoreceptor families and regulates photomorphogenesis (Cluis et al., 2004), we examined the effect of light on hy5-215 tolerance to Pi deficiency. When the seedlings were grown in Pi-deficient conditions under continuous white light, WT and hy5-215 PR lengths were $28 \%$ and $46 \%$ of PR lengths under Pi-sufficient conditions, respectively (Figure 7A). Under continuous dark, there were no significant differences in PR growth between WT and hy5-215 (Figure 7B). These results, together with the results from longday treatments ( $16 \mathrm{~h}$ light/ $8 \mathrm{~h}$ dark; Figure 4B), indicate that increased light irradiation time inhibits Arabidopsis PR growth in Pi-deficient conditions. Therefore, light may play a role in hy5215 tolerance to Pi deficiency.

To better understand light effects on Pi-deficiency tolerance, Arabidopsis plants were grown under continuous blue (B), red (R), and far-red (FR) light. PR growth was inhibited by Pi deficiency in the WT under continuous B light (Figures 8A, B). In contrast, the same level of inhibition by Pi deficiency under B light was not observed in hy5-215. Interestingly, PR growth was not inhibited by Pi deficiency in the WT and all tested mutants when grown under continuous R and FR irradiation (Figures 8A, C). These results indicate that the root tolerant phenotype of hy5-215 to Pi deficiency is negatively regulated by $\mathrm{B}$ light. To further confirm this finding, the B light receptor mutants, cry1 cry 2 and phot1 phot2, were examined under Pi deficiency (Fankhauser and Chory, 1999; Kinoshita et al., 2001). Indeed, a tolerant phenotype to Pi deficiency was found in these two mutants (Figure 9). Therefore, the tolerance of hy5-215 to Pi deficiency likely results from blockage of $\mathrm{B}$ light responses, and the tolerance mechanism may be related to enhancement of internal Pi recycling or utilization efficiency but not external Pi acquisition due to the observation of hy5-215 tolerance on Pi-free condition. Our findings may provide valuable insights for
A

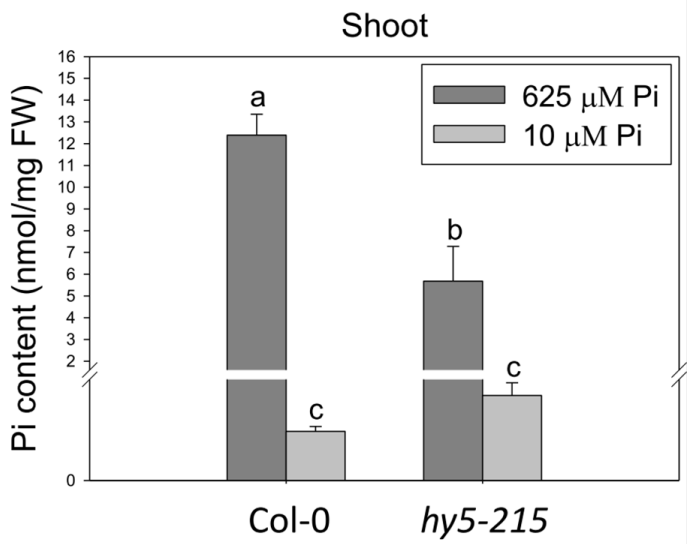

B



C

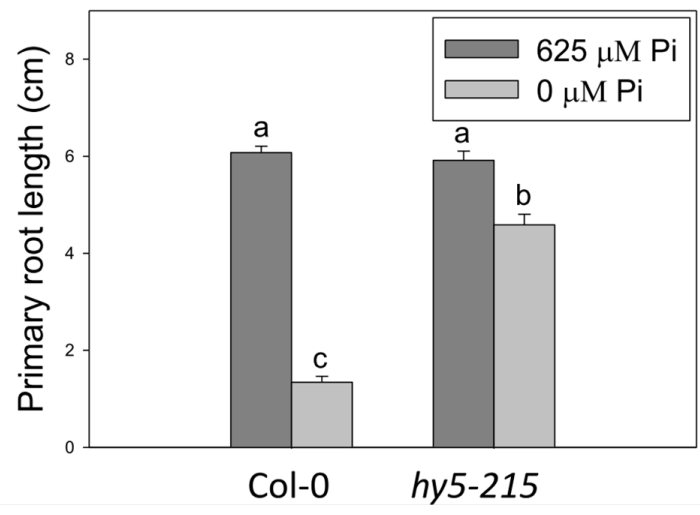

FIGURE 6 | Pi content in wild-type and mutant seedlings in response to $\mathrm{Pi}$ treatment. (A) Soot Pi content in Col-0 and hy5-215. (B) Root Pi content in Col-0 and hy5-215. (C) PR length in Col-0 and hy5-215 when Pi was sufficient or absent. The seedlings were grown on 1/2 MS medium with 625, 10 , or $0 \mu \mathrm{M}$ Pi for 10 days. Data represent means \pm SE of four independent experiments. Different letters above the bars indicate statistically significant differences among the means based on Two-way ANOVA followed by Fisher's LSD tests $(P<0.05)$. 
A

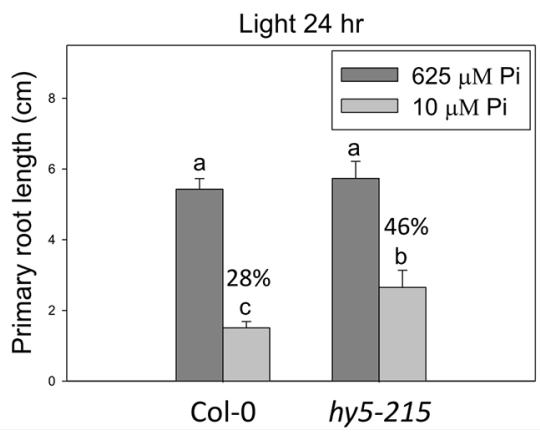

$\mathrm{B}$

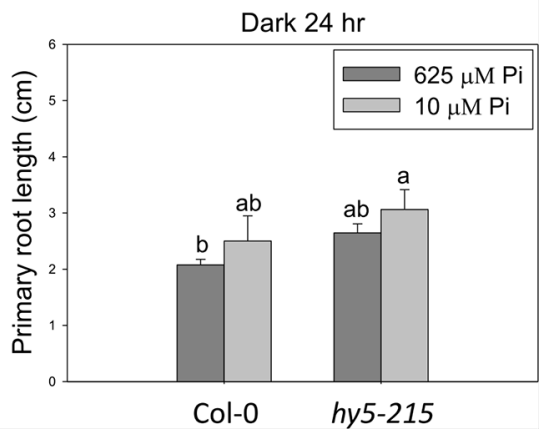

FIGURE 7 | Effect of light on Pi-deficiency tolerance in Arabidopsis. The seedlings were grown on 1/2 MS media with 625 or $10 \mu \mathrm{M}$ Pi under continuous light (A) or dark (B) treatments. The PR length was measured after 10 days of growth. Data represent means \pm SE of four independent experiments. Different letters above the bars indicate statistically significant differences among the means based on Two-way ANOVA followed by Fisher's LSD test $(P<0.05)$.

developing Pi deficiency-tolerant crops in the future. Furthermore, light quality-regulated responses to Pi deficiency may allow indoor plant growers to reduce $\mathrm{Pi}$ fertilizer application through proper illumination.

\section{Identification of Possible Candidate Genes Responsible for Pi-Deficiency Tolerance in 35S:BBX32-SRDX and hy5-215}

To determine the Pi-deficiency tolerance mechanism of hy5-215 and 35S:BBX32-SRDX, we performed several microarray experiments using mRNAs from shoots and roots. The transcriptomic analysis indicates a large number of genes show similar expression pattern between hy5-215 and 35S:BBX32SRDX samples (Supplementary Figure 11 and Tables 1-3). Consistent with previous reports, the well-known PSI genes were up-regulated in the WT under Pi deficiency. However, the expression levels of most PSI genes were significantly lower in hy5-215 and 35S:BBX32-SRDX, including genes encoding highaffinity Pi transporters, ribonucleases, acid phosphatases, lipid remodeling, and anthocyanin synthesis enzymes (Supplementary Table 1). Previously reported Pi deficiencyresponsive TF genes in Arabidopsis mainly belong to the MYB and WRKY families (Rubio et al., 2001; Bustos et al., 2010; Yeh and Ohme-Takagi, 2015). In this study, various TF genes, including MYB, WRKY, AP2/ERF, bHLH, C2H2ZnF, and $M A D S$-box, were up-regulated or down-regulated in hy5-215 and 35S:BBX32-SRDX under Pi-deficient conditions (Supplementary Table 2), suggesting their possible roles in the Pi deficiency tolerance. Liu et al. (2017) have reported that HY5 negatively regulates expression of $P H R 1$ and its downstream PSI genes, and hy5 mutation increases $\mathrm{Pi}$ and anthocyanin contents. According to their data, the longer root phenotype of hy5 under phosphate starvation may result from the increased PSRs and Pi content. Although their root phenotypes of hy5 are similar to our results, PSI gene expression and PSRs are different between two studies. The expression of PSI genes and anthocyanin content were lower in the hy5-215 mutant in our study (Supplementary Figures 3-5 and Table 1), which is consistent with previous reports that the expression of anthocyanin biosynthesis genes and anthocyanin accumulation are reduced in hy5 (Lee et al., 2007; Jeong et al., 2010; Shin et al., 2013). In addition, they reported an increase in total $\mathrm{Pi}$ content in hy5-215 seedlings under Pi sufficient condition; however, we found that free $\mathrm{Pi}$ content is significantly lower in hy5-215 shoots (Figure 6). Our results clearly show that the hy5-215 tolerant phenotype to $\mathrm{Pi}$ starvation is unlikely to be related to external Pi uptake because of similar growths of hy5-215 on Pi-deficient and Pi-free conditions (Figure 6C). Further study is required to address whether these inconsistencies result from different growth conditions or plant tissues selected for the experiments. For example, we performed all the experiments by using both shoot and root samples, but Liu et al. (2017) used whole seedlings. Many genes or metabolisms show distinct expression patterns in specific tissues or organs; therefore, analysis using a mixture of shoot and root samples may not detect their real state in each organs. Sakuraba et al. (2018) reported that HY5 can activate high-affinity Pi transporter genes including PHT1;1, PHT1;4, PHT1;5, PHT1;8, and PHT1;9. Overexpression of HY5 promotes Pi uptake activity, while loss-of-function of HY5 reduces the activity. These results to some degree support our findings but not Liu's (Liu et al., 2017). However, Sakuraba et al. (2018) also used Arabidopsis whole seedlings as materials. Their results may mainly reflect the status of the PHT1 gene expression and Pi content in shoots because the biomass of shoot is much higher than that of root (around 3-5 times in our study). As shown in Supplemental Figure 7, the expression patterns of PHT1;1, PHT1;4, PHT1;5, PHT1;8, and PHT1;9 in hy5-215 shoots are similar to the results shown by Sakuraba et al. (2018). However, the expression of those genes in hy5-215 roots are opposite. It suggests that the regulation of the PHT1 genes may be different in shoots and roots. In addition, they concluded that the HY5mediated activation of $\mathrm{Pi}$ uptake is red-light-dependent. 
A
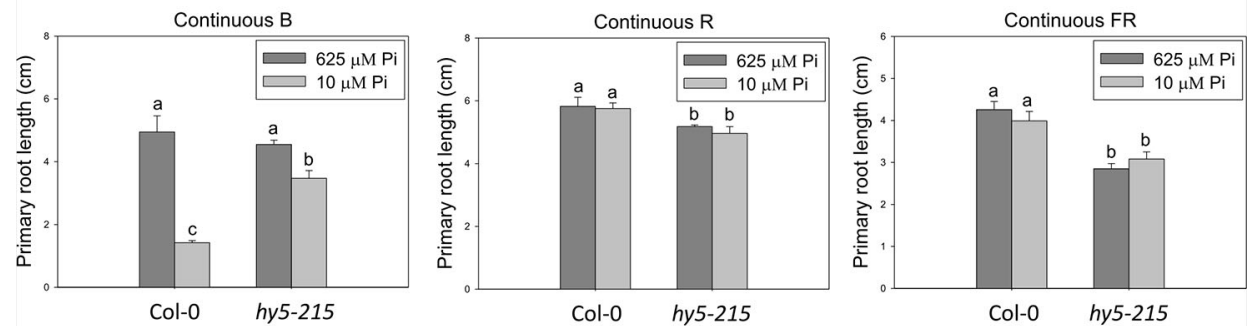

B

Continuous B



C

Continuous $\mathrm{R}$


FIGURE 8 | Effect of light quality on primary root length in Arabidopsis. The Col-0 and hy5-215 seedlings were grown on $1 / 2$ MS media with 625 or $10 \mu \mathrm{M}$ Pi under continuous blue (B), red (R), or far red (FR) light treatments, respectively. The PR length was measured after 10 days of growth (A). The Col-0, hy5-215, slr, and hy5215slr-1 seedlings grown on Pi-sufficient and Pi-deficient media under continuous B (B) and R (C) light treatments. Data represent means \pm SE of four independent experiments. Different letters above the bars indicate statistically significant differences among the means based on Two-way ANOVA followed by Fisher's LSD test $(P<0.05)$

However, in this study, the HY5-regulated PR growth inhibition is dependent on blue light treatment. The results indicate that light quality may play different roles in regulating different PSRs.

Unexpectedly, a significant number of photosynthesis-related and chlorophyll synthesis genes were down-regulated in roots but not shoots of hy5-215 and 35S:BBX32-SRDX (Supplementary Figure 11 and Table 3). Reduction of photosynthesis is a wellknown PSR due to the reduction of Pi consumption for ATP synthesis (Wu et al., 2003; Kang et al., 2014). Plant roots can accumulate chlorophyll and turn green under light illumination. The green roots are supposed to have photosynthetic ability as green leaves (Kobayashi et al., 2012). We therefore considered whether the Pi-deficiency tolerance of hy5-215 is related to down-regulation of photosynthesis-related and chlorophyll synthesis genes, which may induce lower Pi consumption by decreasing photosynthesis in hy5215 roots. GLK1 and GLK2 have been shown to regulate expression of various photosynthetic genes in Arabidopsis roots (Kobayashi et al., 2012; Kobayashi et al., 2013). In addition, it was reported that 
A

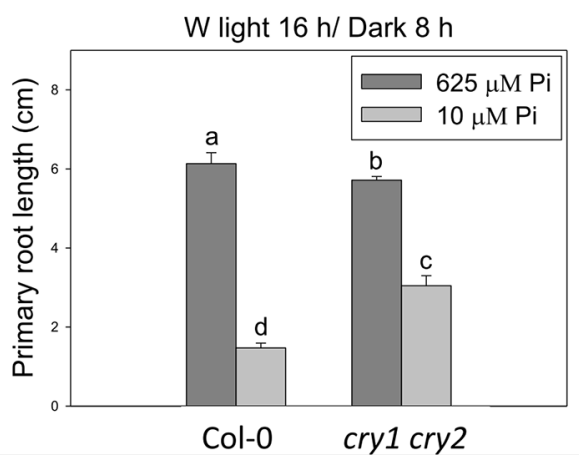

$\mathrm{B}$

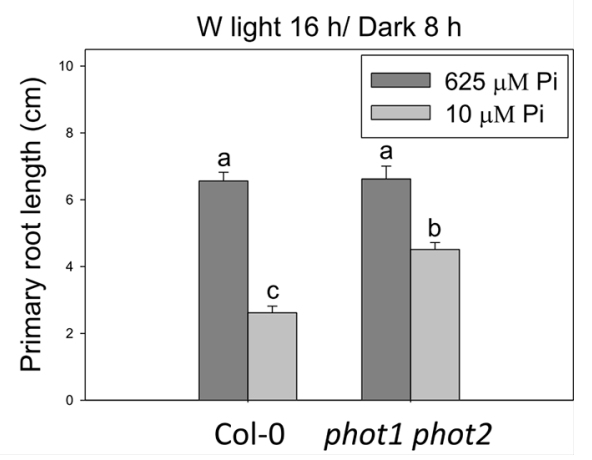

FIGURE 9 | Primary root length of wild-type and blue light receptor mutant seedlings in response to Pi treatment. The blue light receptor mutants, cry1 cry2 (A) and phot1 phot2 (B), were grown on Pi-sufficient and Pi-deficient media under long-day condition (16 h light/8 h dark). The PR length was measured after 10 days of growth. Data represent means \pm SE of four independent experiments. Different letters above the bars indicate statistically significant differences among the means based on Two-way ANOVA followed by Fisher's LSD test $(P<0.05)$.

the roots of 35S:GLK1 accumulate much chlorophyll and are hypersensitive to Pi deficiency (Kang et al., 2014).We thus examined whether the glk mutants also show tolerance to $\mathrm{Pi}$ deficiency (Nagatoshi et al., 2016). The similar PR lengths between WT and glk mutants indicate GLK1 and GLK2 may not be involved in Pi-deficiency tolerance (Supplementary Figures 12A, C). We further investigated the overexpression lines of GLK1 and GLK2 in hy5-215 background (35S:GLK1 hy5-215 and 35S:GLK2 hy5-215), which have a recovered chlorophyll content as WT (Kobayashi et al., 2012). The 35S:GLK1 hy5-215 and 35S:GLK2 hy5-215 plants exhibited longer PR lengths under Pi deficiency similar to hy5-215 (Supplementary Figure 12B), suggesting that tolerance of hy5-215 to $\mathrm{Pi}$ deficiency may not be related to chlorophyll content and photosynthetic activity.

The photosynthetic ability of hy5-215 and WT plants was also compared in shoots, although photosynthetic gene expression in shoots was not significantly different between hy5-215 and WT under both sufficient and deficient conditions. As shown in Supplementary Figure 13, the maximum quantum yield of photosystem II (Fv/Fm) and the actual quantum yield of photosystem II under light (YII) were reduced in the cotyledons of both WT and hy5-215 in response to $\mathrm{Pi}$ deficiency. Although the measurement of Fv/Fm and $\mathrm{Y}_{\mathrm{II}}$ of hy5-215 under Pi sufficient treatment were lower than those of WT, there was no significant difference between WT and hy5-215 in response to $\mathrm{Pi}$ depletion. In addition, $\mathrm{Fv} / \mathrm{Fm}$ and $\mathrm{Y}_{\mathrm{II}}$ in the true leaves of WT and hy5-215 were not affected by our low Pi treatment. These data indicate that the tolerance of hy5-215 to Pi deficiency is not related to photosynthetic ability (Supplementary Figure 13). Enhancement of RNA degradation by ribonucleases or phosphatases is another wellknown mechanism against $\mathrm{Pi}$ deficiency. Therefore, the significant down-regulation of a large number of photosynthesis and chlorophyll biosynthesis genes in hy5-215 roots might, to some degree, contribute to Pi deficiency tolerance through reduction of $\mathrm{Pi}$ consumption for biosynthesis of RNA rather than for photosynthesis.

\section{Identification of Genes Responsible for Blue Light-Regulated Primary Root Growth Inhibition in Response to Pi Deficiency}

Because the PR growth inhibition in response to Pi deficiency only appears under B light illumination, the comparison of root transcriptomes between Arabidopsis WT seedlings grown under $\mathrm{B}$ and $\mathrm{R}$ light treatments was performed to identify candidate genes responsible for Pi deficiency tolerance. Genes encoding enzymes involved in different metabolic pathways were analyzed. Metabolic pathway analysis indicates that numerous class III peroxidase (PRX) genes were significantly down-regulated in response to $\mathrm{Pi}$ deficiency under $\mathrm{R}$ light condition (Supplementary Figure 14). It has been reported that $P R$ growth inhibition in response to $\mathrm{Pi}$ deficiency is mediated by PRX activity at Arabidopsis root tips (Balzergue et al., 2017). Class III PRXs are involved in cell wall tightening by catalyzing crosslinking between some polysaccharides or proteins, subsequently leading to inhibition of cell expansion (Balzergue et al., 2017). Therefore, we next examined the expression levels of the 73 Class III PRX genes in the Arabidopsis genome and identified that 17 of them are Pi deficiency-inducible in three different sets of microarray experiments, including the root transcriptomes of WT corresponding to those of 35S:BBX32SRDX and hy5-215, and the root transcriptome of WT under B light treatment (columns 1-3 in Figure 10). Interestingly, all of these PRX genes were down-regulated or not induced by $\mathrm{Pi}$ deficiency under $\mathrm{R}$ light illumination. The values of $\log _{2}$ fold changes from $R$ to $B$ light $(R / B)$ indicate the PRX genes were 


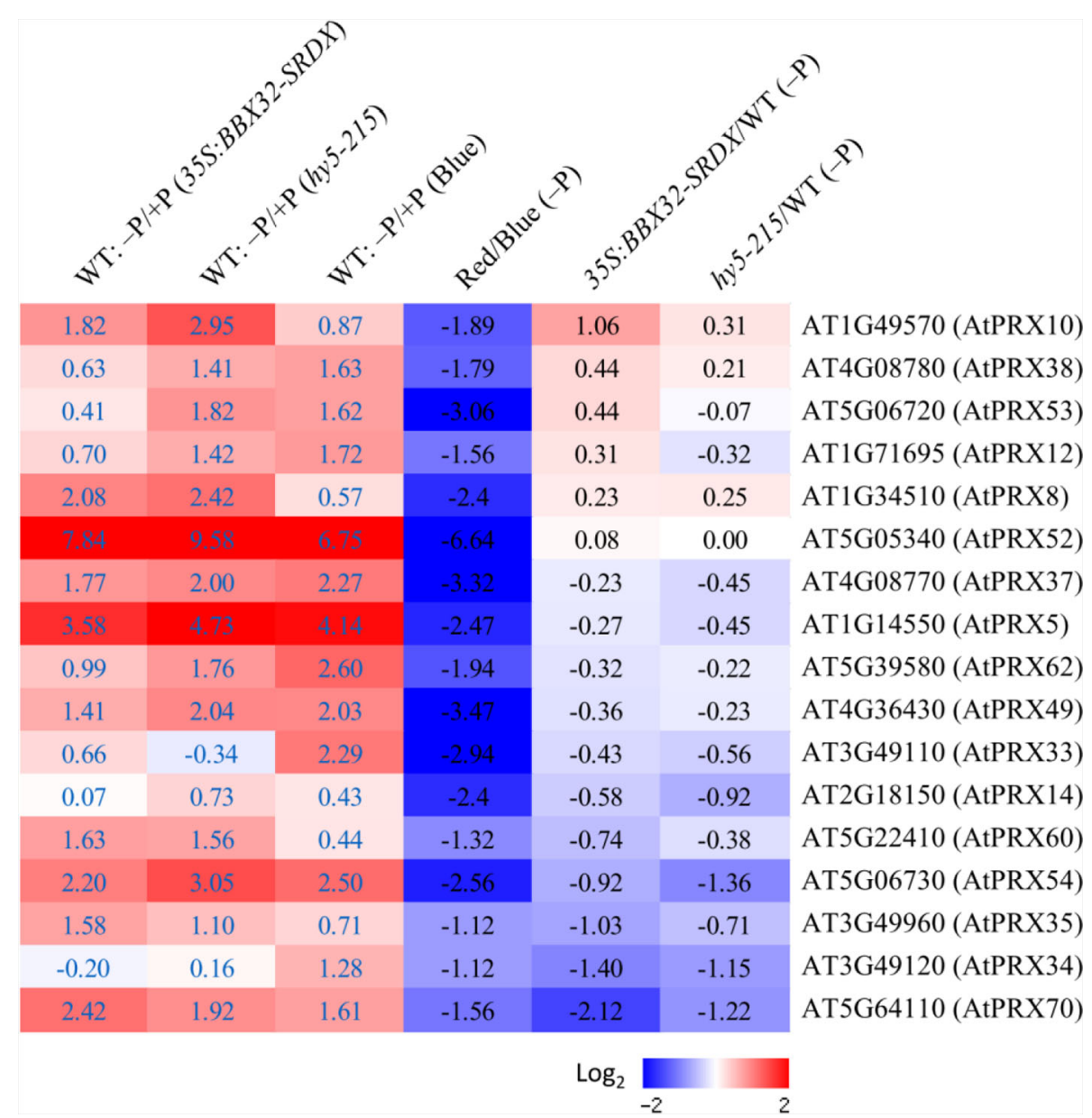

FIGURE 10 | Expression of Pi deficiency-inducible Class III PRX genes under different light treatments. Expression levels of Class III PRX genes were analyzed under different conditions or in different transformants/mutants. The log2 fold change was calculated. Columns 1-3 represent expression levels of PRX genes induced by Pi deficiency in WT roots from different sets of transcriptome experiments (35S:BBX32-SRDX, hy5-215, and B light, respectively). Columns 4 to 6 represent the expression ratio of PRX genes under criteria of R/B, 35S:BBX32-SRDXNT, and hy5-215/WT in response to Pi deficiency.

under-expressed (column 4 in Figure 10). In addition, most of these genes show a similar expression pattern in 35S:BBX32SRDX and hy5-215 when compared to the WT (columns 5 and 6 in Figure 10). These findings imply that the long PR lengths of 35S:BBX32-SRDX and hy5-215 under Pi deficiency may, at least partially, result from lower cell wall stiffness caused by the reduction of PRX gene expression from the blockage of B-light responses. Therefore, the phenotype of 35S:BBX32-SRDX and hy5-215 may represent Pi-deficiency insensitivity but not tolerance. However, in addition to longer PR lengths, the shoot sizes and seedling FWs of 35S:BBX32-SRDX and hy5-215 are bigger and higher, respectively, than those of WT under Pideficient condition. The results suggest potential Pi-deficiency tolerance in 35S:BBX32-SRDX and hy5-215.

In the pathway proposed by Balzergue et al. (2017), Pi deficiency post-transcriptionally activates STOP 1 and subsequently induces $A L M T 1$ expression leading to malate efflux to the apoplast. In turn, $\mathrm{Fe}$ chelation by malate and $\mathrm{Fe}$ redox cycling mediated by LPR1 ferroxidase activity inhibit cell elongation in PR tip through stimulation of peroxidasedependent cell wall stiffening. Although the PR growths and suppression of PRXs in 35S:BBX32-SRDX and hy5-215 are similar to stop 1 and almt1, the expression levels of STOP1 and ALMT1 were not reduced in 35S:BBX32-SRDX, hy5-215, or R/B condition. These results indicate that BBX32 and HY5 do not act upstream of STOP1 and ALMT1 and B light regulates pathways distinct to the STOP1-ALMT1-dependent pathway. Altogether, this study indicates that BBX32 and HY5 are involved in the regulation of PSRs and that $\mathrm{B}$ light may regulate $\mathrm{PR}$ growth inhibition in response to Pi deficiency by the activation of Class III PRX genes. Moreover, a novel mechanism other than the known PSRs may account for the tolerance of 35S:BBX32-SRDX and hy5-215 to Pi deficiency. Further studies, such as metabolome analysis, are required to comprehensively address the roles of BBX32, HY5 and light quality in Pi deficiency responses or tolerance. 


\section{MATERIALS AND METHODS}

\section{Plant Materials and Growth Conditions}

Twenty-thousand T2 seeds of Arabidopsis CRES-T lines were used to screen candidates with altered phenotypes in response to Pi deficiency. The seeds were sterilized by $0.5 \%$ sodium hypochlorite for 10 minutes and then washed with sterile distilled water for at least three times before sowing on $1 / 2$ Murashige and Skoog (MS) media with $10 \mu \mathrm{M} \mathrm{Pi}\left(\mathrm{K}_{2} \mathrm{HPO}_{4}\right)$. The CRES-T seedlings were grown on vertical plates for 10 days. Several phenotypes affected by Pi deficiency including plant size, primary root length, and anthocyanin accumulation were observed or measured.

The surface-sterilized seeds of Arabidopsis thaliana wild type [ecotypes Columbia (Col-0)], mutants (hy5-215, slr-1, hy5-215 slr-1, glk1, glk2, glk1 glk2, cry1 cry2, phot1 phot2), and transformants (35S:BBX32-SRDX, 35S:BBX32, 35S:GLK1 hy5215, and 35S:GLK2 hy5-215) were sown on 1/2 MS agar plates containing $625 \mu \mathrm{M} \mathrm{KH}_{2} \mathrm{PO}_{4}$ (Pi sufficient) or $10 \mu \mathrm{M} \mathrm{KH}_{2} \mathrm{PO}_{4}$ (Pi deficient). Each experiment used 10 plants and was replicated three to four times. The seedlings were grown at $22^{\circ} \mathrm{C}$ and illuminated with $100-125 \mu \mathrm{mol} \mathrm{m} \mathrm{m}^{-2} \mathrm{~s}^{-1}$ white light for 16 hours per day or with blue (B), red (R), and far-red (FR) light for 24 hours. For determination of primary root (PR) length and fresh weight, the seedlings were cultured on vertical and horizontal plates for 10 and 14 days, respectively. The seedlings were then collected for photographs, measurement of PR length and fresh weight, and further experiments.

\section{Quantification of Anthocyanin Content}

The shoots of 10-day-old seedlings were frozen in liquid nitrogen, ground into a powder, and then re-suspended in an extraction buffer containing 45\% methanol and 5\% acetic acid. The supernatant was taken after centrifugation at 12,000 rpm for 10 minutes. Anthocyanin content was calculated by the absorbance at 530 and $637 \mathrm{~nm}$ as described previously (Matsui et al., 2004).

\section{Determination of Acid Phosphatase Activity}

The histochemical staining of acid phosphatase activity was performed according to the method described by $\mathrm{Yu}$ et al. (2012) with some modifications. The roots of 10-day-old seedlings were overlaid with a $0.1 \%$ agar solution containing 0.01\% 5-bromo-4-chloro-3-indolyl phosphate (BCIP). The acid phosphatase activity indicated by blue color on the root surface was observed and photographed after 6 to 24 hours.

\section{Determination of Lipid Composition}

Seedlings were grown on $1 / 2 \mathrm{MS}$ medium with $625 \mu \mathrm{M}$ Pi for 10 days and then transferred to $1 / 2 \mathrm{MS}$ medium with $625 \mu \mathrm{M}$ Pi or $10 \mu \mathrm{M}$ Pi for 10 days. Samples were collected and immediately frozen in liquid nitrogen. Lipids were then extracted and analyzed by the method described by Kobayashi et al. (2006).

\section{RNA Isolation, Reverse-Transcription Quantitative PCR (RT-qPCR), and Microarray Analyses}

Total RNA was extracted by using the RNeasy Plant Mini kit (QIAGEN, Hilden, Germany) following the manufacturer's instructions. One microgram $(1 \mu \mathrm{g})$ of total RNA was subjected to first-strand cDNA synthesis using the PrimeScript RT reagent kit (Takara). Quantitative RT-qPCR was performed by the SYBR green method using the ABI7300 real-time PCR system (Applied Biosystems) as described previously (Mitsuda et al., 2005). The UBQ1 gene was used as an internal control. The microarray experiments and the data analysis were conducted by the method described by Mitsuda et al. (2005). Three biological replicates were performed for the microarray studies except for the experiments conducted under blue and red light treatments (two biological replicates), which aim to isolate candidate genes with similar expression pattern to the microarray experiments performed in 35S:BBX32-SRDX and hy5-215. The expression of the Class III PRX candidate genes isolated from the transcriptomes in response to blue and red light treatments was confirmed by the third biological replicates using RTqPCR. For confirming the other transcriptomes, the RT-qPCR data are averages from three or four biological replicates.

\section{Measurement of Photosynthetic Activity}

The maximum quantum yield of photosystem II $\left(\mathrm{F}_{\mathrm{v}} / \mathrm{F}_{\mathrm{m}}\right)$ and actual quantum yield of photosystem II in light $\left(\mathrm{Y}_{\mathrm{II}}\right)$ of cotyledons and true leaves were measured according to the method described by Kobayashi et al. (2013).

\section{Statistical Analysis}

All the experiments were performed in a completely randomized design. Data on root length $(\mathrm{cm})$ and seedling fresh weight $(\mathrm{mg})$ were recorded after growth for 10 and 14 days, respectively. Twoway analysis of variance (ANOVA) and mean comparisons using least significant difference (LSD) tests were conducted. Data represent means of three or four independent experiments. Different letters above bars indicate statistically significant differences $(P<0.05)$.

\section{DATA AVAILABILITY STATEMENT}

Arabidopsis Genome Initiative numbers described in this article are as follows: ACP5 (At3g17790), CHS (At5g13930), DFR (At5g42800), GLK1 (At2g20570), GLK2 (At5g44190), HY5 (At5g11260), IPS1 (At3g09922), LDOX (At4g22880), MGD2 (At5g20410), MGD3 (At2g11810), MYB75 (At1g56650), MYB90 (At1g66390), NPC4 (At3g03530), PHT1;2 
(At5g43370), PHT1;3 (At5g43360), PHT1;4 (At2g38940), PHT1;5 (At2g32830), PHT1;7 (At3g54700), PHT1;8 (At1g20860), PHT1;9 (At1g76430), RNS1 (At2g02990), SLR/ IAA14 (At4g14550), SQD1 (At4g33030), SQD2 (At5g01220) and UF3GT (AT5G54060). The microarray data referred in this study is available from NCBI GEO under the accession number GSE139100.

\section{AUTHOR CONTRIBUTIONS}

$\mathrm{C}-\mathrm{MY}$ and MO-T designed the experiments and coordinated the project. C-MY performed most of the experiments. KK and SF conducted the analysis of lipid composition and photosynthetic activity. NM performed the microarray experiments. HF created the $s l r$ and hy 5 slr mutants, propagated the seeds, and provided valuable comments on the manuscript. C-MY analyzed the data. $\mathrm{C}-\mathrm{MY}$ wrote the manuscript and MO-T revised the manuscript.

\section{FUNDING}

This work was supported by JSPS KAKENHI Grant Numbers 12F02210, 25291055.

\section{REFERENCES}

Balzergue, C., Dartevelle, T., Godon, C., Laugier, E., Meisrimler, C., Teulon, J. M., et al. (2017). Low phosphate activates STOP1-ALMT1 to rapidly inhibit root cell elongation. Nat. Commun. 8, 15300. doi: 10.1038/ncomms15300

Bari, R., Datt Pant, B., Stitt, M., and Scheible, W. R. (2006). PHO2, microRNA399, and PHR1 define a phosphate-signaling pathway in plants. Plant Physiol. 141, 988-999. doi: 10.1104/pp.106.079707

Bayle, V., Arrighi, J. F., Creff, A., Nespoulous, C., Vialaret, J., Rossignol, M., et al. (2011). Arabidopsis thaliana high-affinity phosphate transporters exhibit multiple levels of posttranslational regulation. Plant Cell 23, 1523-1535. doi: 10.1105/tpc.110.081067

Bhosale, R., Giri, J., Pandey, B. K., Giehl, R. F. H., Hartmann, A., Traini, R., et al. (2018). A mechanistic framework for auxin dependent Arabidopsis root hair elongation to low external phosphate. Nat. Commun. 9, 1409. doi: 10.1038/ s41467-018-03851-3

Bustos, R., Castrillo, G., Linhares, F., Puga, M. I., Rubio, V., Pérez-Pérez, J., et al. (2010). A central regulatory system largely controls transcriptional activation and repression responses to phosphate starvation in Arabidopsis. PloS Genet. 6, e1001102. doi: 10.1371/journal.pgen.1001102

Chen, Z. H., Nimmo, G. A., Jenkins, G. I., and Nimmo, H. G. (2007). BHLH32 modulates several biochemical and morphological processes that respond to Pi starvation in Arabidopsis. Biochem. J. 405, 191-198. doi: 10.1042/ BJ20070102

Chen, Y. F., Li, L. Q., Xu, Q., Kong, Y. H., Wang, H., and Wu, W. H. (2009). The WRKY6 transcription factor modulates PHOSPHATE1 expression in response to low Pi stress in Arabidopsis. Plant Cell 21, 3554-3566. doi: 10.1105/ tpc.108.064980

Chiou, T. J., Aung, K., Lin, S. I., Wu, C. C., Chiang, S. F., and Su, C. L. (2006). Regulation of phosphate homeostasis by microRNA in Arabidopsis. Plant Cell 18, 412-421. doi: 10.1105/tpc.105.038943

Cluis, C. P., Mouchel, C. F., and Hardtke, C. S. (2004). The Arabidopsis transcription factor HY5 integrates light and hormone signaling pathways. Plant J. 38, 332-347. doi: 10.1111/j.1365-313X.2004.02052.x

Devaiah, B. N., Karthikeyan, A. S., and Raghothama, K. G. (2007a). WRKY75 transcription factor is a modulator of phosphate acquisition and root

\section{ACKNOWLEDGMENTS}

We thank Professor Kiyotaka Okada (National Institute for Basic Biology, Japan) for providing hy5-215 seeds; Professor Shigo Takagi (Osaka University, Japan) and Professor Hirokazu Tsukaya (The University of Tokyo, Japan) for phot1, phot2, and phot 1 phot 2 seeds; Professor Christian Fankhauser (University of Lausanne, Switzerland) for cry1 cry2 seeds, and Dr. Yukari Nagatoshi (JIRCAS, Japan) for $g l k 1, g l k 2$, and $g l k 1$ glk2 seeds. We also thank Dr. Sumire Fujiwara, Dr. Yukari Nagatoshi, and Dr. Kouki Yoshida for discussion on the research, and Naomi Ujiie, Machiko Onuki, Yukie Kimura, and Sumiko Takahash for technical assistance. We acknowledge the Max Planck Institute of Molecular Plant Physiology to provide the open-source software of MapMan for microarray analysis.

\section{SUPPLEMENTARY MATERIAL}

The Supplementary Material for this article can be found online at: https://www.frontiersin.org/articles/10.3389/fpls.2019.01803/ full\#supplementary-material development in Arabidopsis. Plant Physiol. 143, 1789-1801. doi: 10.1104/ pp.106.093971

Devaiah, B. N., Nagarajan, V. K., and Raghothama, K. G. (2007b). Phosphate homeostasis and root development in Arabidopsis are synchronized by the zinc ?nger transcription factor ZAT6. Plant Physiol. 145, 147-159. doi: 10.1104/ pp.107.101691

Devaiah, B. N., Madhuvanthi, R., Karthikeyan, A. S., and Raghothama, K. G. (2009). Phosphate starvation responses and gibberellic acid biosynthesis are regulated by the MYB62 transcription factor in Arabidopsis. Mol. Plant 2, 4358. doi: $10.1093 / \mathrm{mp} / \mathrm{ssn} 081$

Fankhauser, C., and Chory, J. (1999). Photomorphogenesis: light receptor kinases in plants! Curr. Biol. 9, R123-R126. doi: 10.1016/S0960-9822(99)80078-5

Fukaki, H., Tameda, S., Masuda, H., and Tasaka, M. (2002). Lateral root formation is blocked by a gain-of-function mutation in the SOLITARY-ROOT/IAA14 gene of Arabidopsis. Plant J. 29, 153-168. doi: 10.1046/j.0960-7412.2001.01201.x

Gaxiola, R. A., Edwards, M., and Elser, J. J. (2011). A transgenic approach to enhance phosphorus use efficiency in crops as part of a comprehensive strategy for sustainable agriculture. Chemosphere 84, 840-845. doi: 10.1016/ j.chemosphere.2011.01.062

Gilbert, N. (2009). Environment: the disappearing nutrient. Nature 461, 716-718. doi: $10.1038 / 461716 a$

Gruber, B. D., Giehl, R. F., Friedel, S., and von Wirén, N. (2013). Plasticity of the Arabidopsis root system under nutrient deficiencies. Plant Physiol. 163, 161179. doi: $10.1104 /$ pp.113.218453

Hamburger, D., Rezzonico, E., MacDonald-Comber Petétot, J., Somerville, C., and Poirier, Y. (2002). Identi?cation and characterization of the Arabidopsis PHO1 gene involved in phosphate loading to the xylem. Plant Cell. 14, 889-902. doi: 10.1105/tpc.000745

Hiratsu, K., Matsui, K., Koyama, T., and Ohme-Takagi, M. (2003). Dominant repression of target genes by chimeric repressors that include the EAR motif, a repression domain, in Arabidopsis. Plant J. 34, 733-739. doi: 10.1046/j.1365313X.2003.01759.x

Holtan, H. E., Bandong, S., Marion, C. M., Adam, L., Tiwari, S., Shen, Y., et al. (2011). BBX32, an Arabidopsis B-Box protein, functions in light signaling by suppressing HY5-regulated gene expression and interacting with STH2/ BBX21. Plant Physiol. 156, 2109-2123. doi: 10.1104/pp.111.177139 
Ikeda, M., Fujiwara, S., Mitsuda, N., and Ohme-Takagi, M. (2012). A triantagonistic basic helix-loop-helix system regulates cell elongation in Arabidopsis. Plant Cell 24, 4483-4497. doi: 10.1105/tpc.112.105023

Jeong, S. W., Das, P. K., Jeoung, S. C., Song, J. Y., Lee, H. K., Kim, Y. K., et al. (2010). Ethylene suppression of sugar-induced anthocyanin pigmentation in Arabidopsis. Plant Physiol. 154, 1514-1531. doi: 10.1104/pp.110.161869

Kang, J., Yu, H., Tian, C., Zhou, W., Li, C., Jiao, Y., et al. (2014). Suppression of photosynthetic gene expression in roots is required for sustained root growth under phosphate deficiency. Plant Physiol. 165, 1156-1170. doi: 10.1104/ pp.114.238725

Kinoshita, T., Doi, M., Suetsugu, N., Kagawa, T., Wada, M., and Shimazaki, K. (2001). Phot1 and phot2 mediate blue light regulation of stomatal opening. Nature 414, 656-660. doi: 10.1038/414656a

Kobayashi, K., Masuda, T., Takamiya, K., and Ohta, H. (2006). Membrane lipid alteration during phosphate starvation is regulated by phosphate signaling and auxin/cytokinin cross-talk. Plant J. 47, 238-248. doi: 10.1111/j.1365313X.2006.02778.x

Kobayashi, K., Baba, S., Obayashi, T., Sato, M., Toyooka, K., Keränen, M., et al. (2012). Regulation of root greening by light and auxin/cytokinin signaling in Arabidopsis. Plant Cell 24, 1081-1095. doi: 10.1105/tpc.111.092254

Kobayashi, K., Sasaki, D., Noguchi, K., Fujinuma, D., Komatsu, H., Kobayashi, M., et al. (2013). Photosynthesis of root chloroplasts developed in Arabidopsis lines overexpressing GOLDEN2-LIKE transcription factors. Plant Cell Physiol. 54, 1365-1377. doi: 10.1093/pcp/pct086

Lapis-Gaza, H. R., Jost, R., and Finnegan, P. M. (2014). Arabidopsis PHOSPHATE TRANSPORTER1 genes PHT1;8 and PHT1;9 are involved in root-to-shoot translocation of orthophosphate. BMC Plant Biol. 14, 334. doi: 10.1186/ s12870-014-0334-z

Lee, J., He, K., Stolc, V., Lee, H., Figueroa, P., Gao, Y., et al. (2007). Analysis of transcription factor HY5 genomic binding sites revealed its hierarchical role in light regulation of development. Plant Cell 19, 731-749. doi: 10.1105/ tpc. 106.047688

Lei, M., Liu, Y., Zhang, B., Zhao, Y., Wang, X., and Zhou, Y. (2011). Genetic and genomic evidence that sucrose is a global regulator of plant responses to phosphate starvation in Arabidopsis. Plant Physiol. 156, 1116-1130. doi: 10.1104/pp.110.171736

Liu, F., Wang, Z., Ren, H., Shen, C., Li, Y., Ling, H. Q., et al. (2010). OsSPX1 suppresses the function of OsPHR2 in the regulation of expression of OsPT2 and phosphate homeostasis in shoots of rice. Plant J. 62, 508-517. doi: 10.1111/ j.1365-313X.2010.04170.x

Liu, Y., Xie, Y., Wang, H., Ma, X., Yao, W., and Wang, H. (2017). Light and Ethylene coordinately regulate the phosphate starvation response through transcriptional regulation of PHOSPHATE STARVATION RESPONSE1. Plant Cell 29, 2269-2284. doi: 10.1105/tpc.17.00268

Matsui, K., Tanaka, H., and Ohme-Takagi, M. (2004). Suppression of the biosynthesis of proanthocyanidin in Arabidopsis by a chimeric PAP1 repressor. Plant Biotechnol. J. 2, 487-493. doi: 10.1111/j.14677652.2004.00094.x

Matsui, K., Umemura, Y., and Ohme-Takagi, M. (2008). AtMYBL2, a protein with a single MYB domain, acts as a negative regulator of anthocyanin biosynthesis in Arabidopsis. Plant J. 55, 954-967. doi: 10.1111/j.1365313X.2008.03565.x

Misson, J., Raghothama, K. G., Jain, A., Jouhet, J., Block, M. A., Bligny, R., et al. (2005). A genome-wide transcriptional analysis using Arabidopsis thaliana Affymetrix gene chips determined plant responses to phosphate deprivation. Proc. Natl. Acad. Sci. U. S. A. 102, 11934-11939. doi: 10.1073/ pnas. 0505266102

Mitsuda, N., and Ohme-Takagi, M. (2009). Functional analysis of transcription factors in Arabidopsis. Plant Cell Physiol. 50, 1232-1248. doi: 10.1093/pcp/ pcp075

Mitsuda, N., Seki, M., Shinozaki, K., and Ohme-Takagi, M. (2005). The NAC transcription factors NST1 and NST2 of Arabidopsis regulate secondary wall thickenings and are required for anther dehiscence. Plant Cell 17, 2993-3006. doi: 10.1105/tpc.105.036004

Nagarajan, V. K., Jain, A., Poling, M. D., Lewis, A. J., Raghothama, K. G., and Smith, A. P. (2011). Arabidopsis Pht1;5 mobilizes phosphate between source and sink organs, and in?uences the interaction between phosphate homeostasis and ethylene signaling. Plant Physiol. 156, 1149-1163. doi: 10.1104/ pp.111.174805

Nagatoshi, Y., Mitsuda, N., Hayashi, M., Inoue, S., Okuma, E., Kubo, A., et al. (2016). GOLDEN 2-LIKE transcription factors for chloroplast development affect ozone tolerance through the regulation of stomatal movement. Proc. Natl. Acad. Sci. U. S. A. 113, 4218-4223. doi: 10.1073/pnas.1513093113

Nakamura, Y. (2013). Phosphate starvation and membrane lipid remodeling in seed plants. Prog. Lipid Res. 52, 43-50. doi: 10.1016/j.plipres.2012.07.002

Niu, Y. F. (2013). Responses of root architecture development to low phosphorus availability: a review. Ann. Bot. 112, 391-408. doi: 10.1093/aob/mcs285

Nussaume, L., Kanno, S., Javot, H., Marin, E., Pochon, N., Ayadi, A., et al. (2011). Phosphate import in plants: focus on the PHT1 transporters. Front. Plant Sci. 2, 83. doi: $10.3389 /$ fpls.2011.00083

Oyama, T., Shimura, Y., and Okada, K. (1997). The Arabidopsis HY5 gene encodes a bZIP protein that regulates stimulus-induced development of root and hypocotyl. Genes Dev. 11, 2983-2995. doi: 10.1101/gad.11.22.2983

Péret, B., Clément, M., Nussaume, L., and Desnos, T. (2011). Root developmental adaptation to phosphate starvation: better safe than sorry. Trends Plant Sci. 16, 442-450. doi: 10.1016/j.tplants.2011.05.006

Pérez-Torres, C. A., López-Bucio, J., Cruz-Ramírez, A., Ibarra-Laclette, E., Dharmasiri, S., Estelle, M., et al. (2008). Phosphate availability alters lateral root development in Arabidopsis by modulating auxin sensitivity via a mechanism involving the TIR1 auxin receptor. Plant Cell 20, 3258-3272. doi: $10.1105 /$ tpc. 108.058719

Plaxton, W. C., and Tran, H. T. (2011). Metabolic adaptations of phosphatestarved plants. Plant Physiol. 156, 1006-1015. doi: 10.1104/pp.111.175281

Poirier, Y., and Bucher, M. (2002). "Phosphate transport and homeostasis in Arabidopsis," in The Arabidopsis Book. Eds. C. R. Somerville and E. M. Meyerowitz (Rockville, MD: American Society of Plant Biologists), e0024. doi: 10.1199/tab.0024

Raghothama, K. G. (2000). Phosphate transport and signaling. Curr. Opin. Plant Biol. 3, 182-187. doi: 10.1016/S1369-5266(00)00062-5

Rose, T. J., Liu, L., and Wissuwa, M. (2013). Improving phosphorus efficiency in cereal crops: Is breeding for reduced grain phosphorus concentration part of the solution? Front. Plant Sci. 4, 444. doi: 10.3389/fpls.2013.00444

Rubio, V., Linhares, F., Solano, R., Martín, A. C., Iglesias, J., Leyva, A., et al. (2001). A conserved MYB transcription factor involved in phosphate starvation signaling both in vascular plants and in unicellular algae. Genes Dev. 15, 2122-2133. doi: 10.1101/gad.204401

Sakuraba, Y., Kanno, S., Mabuchi, A., Monda, K., Iba, K., and Yanagisawa, S. (2018). A phytochrome-B-mediated regulatory mechanism of phosphorus acquisition. Nat. Plants 4, 1089-1101. doi: 10.1038/s41477-018-0294-7

Sato, A., and Miura, K. (2011). Root architecture remodeling induced by phosphate starvation. Plant Signal. Behav. 6, 1122-1126. doi: 10.4161/ psb.6.8.15752

Shin, D. H., Choi, M., Kim, K., Bang, G., Cho, M., Choi, S. B., et al. (2013). HY5 regulates anthocyanin biosynthesis by inducing the transcriptional activation of the MYB75/PAP1 transcription factor in Arabidopsis. FEBS Lett. 587, 15431547. doi: 10.1016/j.febslet.2013.03.037

Thibaud, M. C., Arrighi, J. F., Bayle, V., Chiarenza, S., Creff, A., Bustos, R., et al. (2010). Dissection of local and systemic transcriptional responses to phosphate starvation in Arabidopsis. Plant J. 64, 775-789. doi: 10.1111/j.1365313X.2010.04375.X

Ticconi, C. A., and Abel, S. (2004). Short on phosphate: plant surveillance and countermeasures. Trends Plant Sci. 9, 548-555. doi: 10.1016/ j.tplants.2004.09.003

Wissuwa, M. (2003). How do plants achieve tolerance to phosphorus deficiency? Small causes with big effects. Plant Physiol. 133, 1947-1958. doi: 10.1104/ pp.103.029306

Woo, J., MacPherson, C. R., Liu, J., Wang, H., Kiba, T., Hannah, M. A., et al. (2012). The response and recovery of the Arabidopsis thaliana transcriptome to phosphate starvation. BMC Plant Biol. 12, 62. doi: 10.1186/1471-2229-1262

Wu, P., Ma, L., Hou, X., Wang, M., Wu, Y., Liu, F., et al. (2003). Phosphate starvation triggers distinct alterations of genome expression in Arabidopsis roots and leaves. Plant Physiol. 132, 1260-1271. doi: 10.1104/ pp.103.021022 
Yeh, C. M., and Ohme-Takagi, M. (2015). Transcription factors involved in acid stress responses in plants. Nucleus 58, 191-197. doi: 10.1007/s13237-016-0159-2

Yeh, C. M., Ohme-Takagi, M., and Tsai, W. C. (2017). Current understanding on the roles of ethylene in plant responses to phosphate deficiency. Int. J. Plant Biol. Res. 5, 1058.

Yu, H., Luo, N., Sun, L., and Liu, D. (2012). HPS4/SABRE regulates plant responses to phosphate starvation through antagonistic interaction with ethylene signalling. J. Exp. Bot. 63, 4527-4538. doi: 10.1093/jxb/ers131

Zhou, J., Jiao, F., Wu, Z., Li, Y., Wang, X., He, X., et al. (2008). OsPHR2 is involved in phosphate-starvation signaling and excessive phosphate accumulation in shoots of plants. Plant Physiol. 146, 1673-1686. doi: 10.1104/pp.107.111443
Conflict of Interest: The authors declare that the research was conducted in the absence of any commercial or financial relationships that could be construed as a potential conflict of interest.

Copyright $\odot 2020$ Yeh, Kobayashi, Fujii, Fukaki, Mitsuda and Ohme-Takagi. This is an open-access article distributed under the terms of the Creative Commons Attribution License (CC BY). The use, distribution or reproduction in other forums is permitted, provided the original author(s) and the copyright owner(s) are credited and that the original publication in this journal is cited, in accordance with accepted academic practice. No use, distribution or reproduction is permitted which does not comply with these terms. 